EXEMPLARIa Classica

Journal of Classical Philology

20, 2016, pp. 227-237

ISSN 1699-3225

\title{
LA RECONSTRUCCIÓN DE LAS HISTORIAS DE SALUSTIO*
}

Antonio La Penna - Rodolfo Funari, C. Sallusti Crispi Historiae I: fragmenta 1.1-146, Berlin - Boston, De Gruyter, 2015, 387 pp. ISBN 9783-11-019566-8, 139.95€.

John T. Ramsey, Sallust. Fragments of Histories. Letters to Caesar. Edited and translated by J. T. R., Loeb Classical Library, Cambridge MA - London, Harvard University Press, 2015, 611 pp. ISBN 978-0-674-99686-1, $\$ 26.00 \cdot £ 16.95 \cdot 21.00 €$.

Después de mucho tiempo sin que aparecieran ediciones de las Historias de Salustio han coincidido en el 2015 dos libros excepcionalmente valiosos, herederos de trabajos publicados en un larguísimo período de tiempo y que sin duda marcarán durante largos años la investigación sobre esta obra.

Como es bien sabido, Historias estaba llamada a ser la obra cumbre de Salustio: es lógico suponer que fue la última que escribió, probablemente entre el 40 a. C. o, más verosímilmente, el 39 y el año de la muerte del escritor, en el 36 o el 35. El autor, en plena madurez creativa, abandonó el modelo de la monografía, que tan excelentes resultados le había dado en la Conjuración de Catilina y la Guerra de Jugurta, para abordar el relato de un período histórico completo, concretamente el que empieza tras el fin voluntario de la dictadura de Sila (79), organizándolo analísticamente y alternando la política interna romana con las guerras externas ${ }^{1}$. No se sabe en realidad cuál sería el término proyectado de la obra: los últimos sucesos identificables entre los fragmentos del libro V datan del 67 a. C., pero son muchos los que piensan que Salustio pudo haber tenido la intención de extenderse hasta el 64 y enlazar así con Catilina. En todo caso la muerte del escritor dejó inconclusa la obra y, de los cinco libros de los que tenemos noticia, el último, seguramente por esta misma razón, es el más pobremente representado. Doce años, por tanto, en los que se desarrollan acontecimientos trascendentales para Roma: la revuelta de Lépido, las guerras contra Mitrídates y contra los piratas, que se dilatan

* Este artículo se inscribe en el proyecto FFI 2014-56798-P del Ministerio de Educación y Ciencia. Utilizo sistemáticamente la numeración de Maurenbrecher para los fragmentos de Historias y, en su caso, además, las de McGushin, Ramsey o La Penna-Funari.

${ }^{1}$ Cf. n. 3, además de A. La Penna, "Per la ricostruzione delle Historiae di Sallustio", Studi italiani di filologia classica 35, 1963, 19-20; Sallustio e la rivoluzione romana, Milano 1968, 337-8. 
año tras año, la rebelión de Sertorio en Hispania, el levantamiento de los esclavos dirigidos por Espartaco y el ascenso imparable de Pompeyo, que se apoya en la resolución de varios de estos conflictos.

Salustio ha sido un autor universalmente leído y admirado a lo largo de todas las épocas y sus obras no han dejado de citarse, gracias a lo cual tenemos al menos cierta idea de sus Historias. Pero el caso es que las copias de estas, por razones que en realidad se nos escapan - iquizá por ser mayor que las demás o por su carácter de obra inacabada?-, debieron ir disminuyendo paulatinamente en la Antigüedad tardía y principios de la Edad Media ${ }^{2}$. El que pudo ser con bastante probabilidad el último códice íntegro de Historias, el Floriacensis (Fleury), del siglo V, llegó hasta el siglo VII: si hubiera alcanzado el renacimiento Carolingio, podríamos estar leyendo actualmente el libro completo, pero en ese momento lo destrozaron para aprovecharlo como material de encuadernación y reutilizarlo en parte para escribir otras obras ${ }^{3}$. A partir de ese momento y con la excepción de los miserables restos de este manuscrito, los testimonios directos de Historias se limitan a tres papiros ${ }^{4}$ y el códice Vaticanus Latinus 3864, del siglo IX, el más importante, gracias al cual conocemos cuatro discursos y dos cartas de la obra 5 .

Aunque los fragmentos más extensos son los procedentes de la trasmisión directa, no suponen más que una mínima parte del número total; el resto de los más de quinientos testimonios viene de citas de otros autores, especialmente gramáticos y escoliastas. Muchos de estos trasmiten el número del libro de Historias de donde están tomados los textos y no es extraño que el tema por el que copian la cita o la declaración expresa del autor proporcionen datos relevantes sobre el contexto original. Pero en otros casos ni se conoce con total seguridad el libro ni la materia. Y naturalmente hay ocasiones en las que ni siquiera se menciona la obra. Incidentalmente, se trata de textos muy dispares, que van desde varios párrafos a apenas una palabra.

Así pues, ordenar todos estos fragmentos tratando de darles un sentido y una interpretación coherente para producir al menos una imagen de la obra

${ }^{2}$ Aunque los gramáticos y escoliastas entre los siglos III y VI no dejan de proporcionar testimonios de Historias, si bien más de uno pudo ser de segunda mano y no de la lectura directa del libro. Sobre estos y todos los demás autores que trasmiten citas véase la introducción de La Penna 2015, 12-35.

${ }^{3}$ Cf. H. Bloch, "The Structure of Sallust's Historiae: The Evidence of the Fleury Manuscript", en S. Prete, ed., Didascaliae: Studies in honor of Anselm Albareda, New York 1961, 61-76. También es importante G. Perl, "Der alte Codex der «Historiae» Sallusts", Bulletin d'information de l'Institut de Recherche et d'Histoire des Textes 15, 1967/68, 29-38, y el excelente resumen de Ramsey 2015, XV-XVIII.

${ }^{4}$ El Rylands de Manchester III 473, procedente de Oxirrinco y escrito quizá entre los siglos II y III; el de Viena (Lat. 117), que data del siglo IV y conserva texto de 1.107 y 136, y otro, del mismo rollo que el de Rylands y conservado en Oxford (Sackler Library; 68 6B.20/L [10-13] a), que trasmite 2.7, cf. R. Funari, Corpus dei papiri storici greci e latini. Parte B: Storici latini. 1. Autori noti. Vol. 2: Caius Sallustius Crispus, Pisa-Roma 2008.

${ }^{5}$ Además de contener, entre otras cosas, discursos y cartas de las demás obras de Salustio. 
original es una tarea ciclópea que, sin embargo, ha atraído a varias generaciones de filólogos; sería injusto no reconocer que por lo menos parcialmente se ha cumplido su objetivo. Aunque las primeras impresiones de fragmentos de Historias se dan pronto en las ediciones completas de Salustio ${ }^{6}$, la que abre el camino para el estudio verdaderamente filológico es la recopilación de Carrión ${ }^{7}$. Tras significativos avances tanto en el estudio en sí de Historias como en el de los autores que trasmiten las citas, se publican ya en el siglo XVIII la edición comentada de Kortte (= Cortius, Lipsiae 1724) y el increíble libro de De Brosses ${ }^{8}$, que a pesar de serias deficiencias y de su peculiar concepción, ha merecido que se le continúe citando hasta hoy en día9 . Ya en el siglo XIX se suceden varias ediciones alemanas que, al menos en parte, mantienen todavía su vigencia: Ramsey, por ejemplo, proporciona las numeraciones de Dietsch y Kritz ${ }^{10}$. La culminación de todas estas es la de Maurenbrecher ${ }^{11}$, que señala el principio y el fundamento de las investigaciones modernas y que, por más que haya que corregir y enmendar, resulta imprescindible en la actualidad.

Tras casi setenta años de dominio absoluto en su campo, La Penna, en un trascendental artículo, realizó una revisión completa de la edición de Maurenbrecher señalando el que indudablemente es su mayor defecto ${ }^{12}$ : dar por cierta la colocación de fragmentos muy inseguros en el contexto de cada uno de los libros, cuando, siendo honrado, no deberían haber pasado de los textos incertae sedis los que se han trasmitido sin número de libro y del final de cada uno de ellos los que están localizados, pero no proporcionan información suficiente para saber a qué se refieren ${ }^{13}$. Por poner un par de ejemplos, hay que dejarse llevar por un optimismo a toda prueba para fijar en el libro III como número 41 este fragmento: paululum requietis militibus.

${ }^{6}$ Útil historia de las ediciones y estudios de la obra en La Penna, Funari 2015, 37-42.

${ }^{7}$ L. Carrio, C. Sallusti Crispi Historiarum libri VI, Antuerpiae 1573. El sexto libro está formado por un solo fragmento, el 1.100, que los manuscritos de Nonio sitúan en el XI (!). Contrariamente a lo que da a entender La Penna, Carrión situó al final y aparte, no en el VI, todos los fragmentos sin número de libro.

${ }^{8}$ Charles de Brosses, Histoire de la République Romaine dans le cours du VIIe siècle par Sallust, 3 vols., Dijon 1777, en el que inserta los fragmentos traducidos al francés - muchos en más de un lugar- en una historia continua de la época.

${ }^{9}$ Maurenbrecher, por ejemplo, sistemáticamente, y a partir de este sus ideas han llegado hasta la actualidad.

${ }^{10}$ F. Kritz, Gai Sallusti Crispi opera quae supersunt. Vol. III: Historiarum fragmenta, Lipsiae 1853 - «Il Kritz è probabilmente colui al que la ricostruzione delle Historiae deve di più» (La Penna 1963 [n. 1],7)—; R. Dietsch, Gai Sallusti Crispi opera quae supersunt. Vol. II: Historiarum reliquiae, Lipsiae 1859.

${ }^{11}$ B. Maurenbrecher, C. Sallusti Crispi Historiarum reliquiae. Fasciculus I: Prolegomena, Lipsiae 1891; C. Sallusti Crispi Historiarum reliquiae. Fasciculus II: Fragmenta, Lipsiae 1893.

${ }^{12}$ La Penna 1963 (n. 1), 5-6.

${ }^{13}$ Todos los editores modernos dejan para el final de cada libro, en una especie de cajón de sastre, aquellos textos que están debidamente localizados, pero cuyo tema se ignora o, por lo menos, no puede precisarse con mediana seguridad. 
Maurenbrecher lo conectó con el $40-U t$ sustinere corpora plerique nequeuntes arma sua quisque stans incumberet (Arus. gramm. 263; Serv. Aen. 9.227) - y Plu. Luc. 11 para aventurar que se refiere igualmente a las penalidades padecidas por los soldados de Luculo. Pero Servio, en su comentario a Virgilio, Bucólicas 8.4, afirma únicamente que pertenece a las Historias de Salustio y, con toda lógica, tanto McGushin ${ }^{14}$ como Ramsey ${ }^{15}$ lo relegan a los de localización incierta. Otro fragmento trasmitido sin indicación de libro es el que aparece como 54 del libro IV: canina, ut ait Appius, facundia exercebatur ${ }^{16}$. Como Lactancio aplica esta expresión a Cicerón, Maurenbrecher relacionó el fragmento con los discursos contra Verres. Naturalmente, esto es completamente arbitrario: antes, por ejemplo, Kritz y Dietsch creyeron que se trataba de la feroz oratoria del tribuno Sicinio (Plu. Crass. 7.8) y lo colocaron en el libro II ${ }^{17}$, y con posterioridad La Penna ${ }^{18}$ propuso encajarlo en la revisión de la oratoria que tenía lugar en el prefacio del libro I, y así lo ha acabado editando ${ }^{19}$. Más razonables, tanto McGushin 1994 como Ramsey 2015 lo dejan en los fragmentos incertae sedis, los números 27 y 33 respectivamente.

Por otra parte, era obvio que la crítica avanzaba y habían surgido algunas fuentes que Maurenbrecher no pudo conocer, como, por ejemplo, el papiro Rylands ${ }^{20}$, así que la necesidad de una nueva edición era imperiosa. Sin embargo, esta se ha retrasado hasta la publicación de los libros objeto de esta reseña. En los años noventa del pasado siglo aparecieron dos obras imprescindibles hoy en día pero que de ninguna manera podían relevar a la ya centenaria recopilación de Maurenbrecher. Por una parte, Funari, el coautor de la edición del libro I, daba a la estampa un comentario completo de los fragmentos de Historias trasmitidos por fuentes indirectas, atento por supuesto a la colocación de los textos, aunque no la alteraba, y especialmente a cuestiones lingüísticas y literarias ${ }^{21}$. Más ambiciosa ciertamente era la edición de McGushin de toda la obra con un amplio y en estos momentos indispensable comentario histórico, que por primera vez no solo cuestionaba la ordenación

${ }^{14}$ Con el número 19, cf. McGushin 1994 (n. 22), II, 222-3. Véase también el natural escepticismo de La Penna 1963 (n. 1), 42.

${ }^{15}$ Ramsey 2015.

${ }^{16}$ Non. p. 60; Lact. inst. 7.18.26.

${ }^{17}$ Kritz 1853 (n. 10), 150-1; Dietsch 1859 (n. 10) 42.

18 "Una polemica di Sallustio contro l'oratoria contemporanea?", RFIC 101, 1973, 88-91.

${ }^{19}$ Con el número 1.3, cf. La Penna, Funari 2015, 121-2.

${ }^{20} \mathrm{C}$. H. Roberts, Catalogue of the Greek and Latin Papyri in the John Rylands Library, Manchester. Volume III: Theological and Literary Texts, Manchester 1938.

${ }^{21}$ R. Funari, C. Sallusti Crispi Historiarum fragmenta, 2 vols., Amsterdam 1996. Existían en esos momentos comentarios más que solventes de los fragmentos mayores, es decir, los que omite Funari: V. Paladini, C. Sallusti Crispi orationes et epistulae de Historiarum libris excerptae, Bologna ${ }^{2}$ 1968; E. Pasoli, Le Historiae e le opere minori di Sallustio, Bologna $1974^{3}(=1965)$. 
de Maurenbrecher, sino que proponía una nueva ${ }^{22}$. La gran desventaja de este libro, que impidió que se convirtiera en edición de referencia, es que contaba con traducción al inglés, pero no con texto latino ni, consiguientemente, con observaciones sobre trasmisión y lengua acordes con la importancia de la materia.

Este es el contexto en el que surgen los libros que aquí se comentan. La Penna ha venido estudiando a Salustio desde los años cincuenta y entre sus publicaciones se contaban ya antes de esta aportaciones esenciales. Funari, por otra parte, ha estudiado tanto los fragmentos de Historias, como ya se ha visto, como la trasmisión papirácea. A Ramsey se le debe tanto un comentario sobre la Conjuración de Catilina, que se ha convertido en modelo $^{23}$, como el anterior volumen de Salustio en la Loeb, renovación del volumen de Rolfe, pero que, en realidad, es una nueva y magnífica edición ${ }^{24}$, de la que el presente libro es la continuación natural.

Las dificultades de una edición de Historias se pueden resumir fundamentalmente en cuatro. Quizá la más liviana sea la existencia de una serie de textos dudosos o espurios: Maurenbrecher identificó solamente seis ${ }^{25}$, mientras que Ramsey tiene cincuenta y cinco. Aunque las divergencias son importantes, sobre todo si se tiene en cuenta que más de uno figura en una edición entre los falsos y en la otra está perfectamente situado en un libro, ni son demasiados textos ni demasiado significativos. Pero ya se puede apreciar en esto el creciente escepticismo que diferencia principalmente a Maurenbrecher de los editores más modernos.

La segunda cuestión es la distribución en un libro de los temas o, como escribía Maurenbrecher, de los capítulos que trataba. En algunos casos se puede invocar la ayuda de algún autor como Julio Exuperancio o las Períocas de Tito Livio; en otros, el editor se mueve por criterios mucho más inseguros. Hay enormes diferencias en el tratamiento que se hace de cada libro en los diversos autores: en el orden en el que aparecían las grandes cuestiones en el libro I se observa una contundente unanimidad en Maurenbrecher, Ramsey y La Penna-Funari ${ }^{26}$, aunque, por supuesto, la colocación de fragmentos concretos presente divergencias. En el libro III, en cambio, las diferencias entre Maurenbrecher y Ramsey se multiplican: este último presenta el primer capítulo de la guerra sertoriana, que divide en dos partes, por delante de la

${ }_{22}^{2}$ P. McGushin, Sallust, The Histories, 2 vols., Oxford 1992-1994.

${ }^{23}$ J. T. Ramsey, Sallust's Bellum Catilinae. Edited, with Introduction and Commentary, Oxford $2007^{2}(=1984)$.

${ }^{24}$ J. C. Rolfe, J. T. Ramsey, Sallust: The War with Catiline. The War with Jugurtha. Translated by J. C. R. revised by J. T. R., Cambridge MA-London 2013; véase la reseña en ExClass 18, 2014, 257-60.

${ }^{25}$ A continuación añade un apéndice De locis falso antea pro fragmentis historiarum Sallustianarum habitis (pp. 210-12).

${ }^{26}$ Véanse respectivamente las páginas 1-2; 47-8; 2-3. 
mitridática, que se organiza en tres series de fragmentos rematados al final del libro por el excurso sobre la geografía del Ponto. Aunque en general parecen razonables muchas de las correcciones de Ramsey, estrechamente dependientes de McGushin en este sentido, da la impresión de que la incertidumbre en la mayoría de los detalles es inevitable.

Colofón necesario del apartado anterior es la disposición de los fragmentos que, aunque estén claramente identificados en las fuentes como pertenecientes a un libro, son muy difíciles de situar. Por poner un ejemplo, que se podría multiplicar muy fácilmente, véase el 80 del libro I según Maurenbrecher: fugam maturabat. (Arus. gramm. 374) ${ }^{27}$. Podría tratarse, por supuesto, de la huida de Lépido tras su derrota ${ }^{28}$ pero caben otras posibilidades igualmente verosímiles dentro del mismo libro I, cf. McGushin 1992 (n. 22), I, 148-9; La Penna-Funari 2015, 295-6.

Pero si estas últimas disputas afectan a la disposición interior de los libros y naturalmente no carecen de importancia, quizá el problema más arduo de todos es la colocación de los fragmentos que se han transmitido sin número de libro. Desde los primeros editores, que, como Carrión, prefirieron relegarlos al final sin pronunciarse sobre el lugar en el que se debían insertar, ha habido una creciente tendencia que culminó en Maurenbrecher, aunque tuvo ya un gran impulso en Kritz, a tratar de distribuir todos los textos por libros. Y así Maurenbrecher reconoce solo treinta y ocho fragmentos incertae sedis. Para Ramsey, en cambio, son sesenta y nueve. Y probablemente esta cifra debería aumentar.

Por consiguiente, los problemas a la hora de ordenar los fragmentos y reconstruir en lo posible al menos el armazón de Historias son de muy distinto calado, tan variados como los mismos textos que hay que organizar, pero si hay algo que caracteriza a todos los que han estudiado el tema después de Maurenbrecher es la revisión de los distintos episodios dentro de cada libro $y$, sobre todo, el convencimiento de que, si no aparecen más testimonios, son muchos los fragmentos cuya colocación debe calificarse simplemente de dudosa, bien al final de cada libro o bien entre los de localización incierta.

Es en esta situación y cuando no se había publicado todavía una edición capaz de remplazar definitivamente a la de Maurenbrecher cuando surgen en el 2015 el libro de La Penna y Funari sobre el libro I y el de Ramsey, que abarca la totalidad de Historias. El primero de ellos constituye el primer volumen de lo que debería ser una versión actualizada y, hasta donde lo permite la evolución de los estudios filológicos, definitiva de Historias y esto es lo que se declara en la Premessa, aunque ni está siquiera anunciado el siguiente volumen ni se aclara cuándo podría aparecer. Los dos autores se han dividido

${ }^{27}$ McGushin lo presenta como fragmento 68; Ramsey, 70 y La Penna, Funari, 74.

${ }^{28}$ Cf. Flor. epit. 2.11 (= 3.23).6-7; Exup. 6; App. BC 1.107. 
todo el prefacio ${ }^{29}$, La Penna se ha ocupado de los fragmentos de tradición indirecta y Funari de los que contiene el códice Vaticano 3864 -los discursos de Lépido y Filipo- y los papiros - sus 1.101 y 104, correspondientes a los 107 y 136 de Maurenbrecher ${ }^{30}$ - El volumen consta de unos excelentes prolegómenos que se remiten en parte a los de Maurenbrecher ${ }^{31}$ y que tratan de los historiadores y otros escritores de la Antigüedad relacionados con la obra salustiana, los gramáticos y escoliastas (pp. 16-31), las conexiones ajenas a cuestiones lingüísticas, las propias de la Edad Media y el Renacimiento (34-7) y las ediciones (37-42). A estos le siguen capítulos sobre la colección de discursos y cartas del manuscrito Vaticano, la estructura de Historiae, la composición del libro I y unas avvertenze.

Lo más importante del libro naturalmente consiste en la edición de cada uno de los 146 fragmentos $^{32}$ con aparato crítico exhaustivo y relación de todas las fuentes con reproducción del contexto en el que se citan (53-102), traducción italiana (103-17) y amplio comentario con la bibliografía esencial de cada texto (119-354). Rematan el volumen una de las bibliografías más completas que se pueden encontrar ${ }^{33}$, una lista de las ediciones en las que se basan los testimonios y otra con las de los autores citados, las abreviaturas y las inevitables correspondencias entre esta edición, la de Maurenbrecher y la de McGushin. El único e incomprensible fallo que se advierte en el volumen es la falta de un índice de nombres, que en una obra tan excelente debería estar rematado además por un index locorum. Es posible que los autores hayan pensado en proporcionarlos al final de la edición entera, pero teniendo en cuenta que esta necesitará dos o tres libros más cuya aparición podría alargarse durante bastantes años, resulta inexplicable que no lo hayan adjuntado aquí.

Por supuesto, siempre hay alguna pequeña inadvertencia o falta y más en una obra tan densa: alguna inexactitud en la traducción ${ }^{34}$, por ejemplo, o quizá fallos en comentarios, como en 105 (333) o 143 (353), en los que se

29 A La Penna le corresponden Prolegomena, La struttura delle Historiae y La composizione del libro I, y a Funari la Introduzione generale: la silloge di orationes e epistulae dalle Historiae di Sallustio nel codice Vat. lat. 3864 y las Avvertenze.

${ }^{30}$ No aparece, por cierto, 1.83 , que se presta a varias interpretaciones y que tanto McGushin como Ramsey relegan a los fragmentos de localización incierta (12 y 2 respectivamente). Es lógico suponer que La Penna piense colocarlo en este mismo lugar.

${ }^{31} 1891$ (n. 11).

${ }^{32}$ En Maurenbrecher eran 153, en Ramsey 139.

${ }^{33}$ Apenas se pueden encontrar aportaciones valiosas que no se consignen. Yo citaría, por ejemplo, J. A. Rosenblitt, "Sallust's Historiae and the Voice of Sallust's Lepidus", Arethusa $46.3,2013,447-70$, tanto en la bibliografía como a propósito del discurso de Lépido, aunque quizá sea demasiado reciente. También hubiera merecido la pena ver la tesis de C. J. Burnand: Roman Representations of the Orator during the Last Century of the Republic, Diss. University of Oxford, Oxford 2000.

${ }^{34}$ En 1.16 (p. 104), correspondiente a 1.12 Maurenbrecher, no se traduce quia praesentia defendebat. 
omiten hipótesis para su interpretación ${ }^{35}$. También, dentro del discurso de Lépido (53), hubieran merecido alguna observación adicional Cimbricam (17) o ancilla $(21)^{36}$, y en el de Filipo, la alusión a la gens Aemilia (p. 251).

Por otro lado, no sé si hubiera sido útil consultar las ediciones antiguas o partir de Maurenbrecher, como se suele hacer; probablemente esta última opción es la más sensata, pero de esta forma se han transmitido también algunas imprecisiones: no fue sugerencia de Kritz, sino de Maurenbrecher que el fragmento $55(59 \mathrm{M})$ pudiera referirse a Sila (p. 224), mientras que la hipótesis de que la ciudad que aparece en $40(40 \mathrm{M})$ es Cástulo (Jaén) se debe a Linker ${ }^{37}$.

No es fácil dar cuenta del excelente trabajo que representa este libro. Sin duda será, cuando llegue a completarse, la edición que remplace a la de Maurenbrecher tanto por la cantidad de información que aduce en la edición de los textos y en el comentario como por lo atinado en general de estos. Quizá en su estructura general y de acuerdo con esta idea de obra total hubiera merecido una introducción todavía mayor que hubiera abordado con la suficiente profundidad las cuestiones históricas, inexcusables en la recomposición de la Historias, aunque las presentaciones de diversos fragmentos, sobre todo de los discursos, palían en parte esta falta.

La edición de Ramsey es lógicamente muy diferente. Se trata de la colección Loeb, por lo que debe contar con texto latino acompañado de un aparato crítico reducido y traducción inglesa anotada. En este caso y debido a las especiales circunstancias de estos textos tiene algunas peculiaridades que la distinguen de la mayoría de los volúmenes de la serie: en primer lugar presenta, bajo el texto latino y antes de las notas críticas, la fuente del fragmento con una breve explicación en inglés de las razones para citarlo. En la página opuesta y antes de la traducción se encuentra, en letra cursiva, una declaración, en general suficientemente amplia, de las circunstancias a las que aluden los fragmentos con alguna noticia sobre las hipótesis más plausibles para su interpretación. De esta forma, Ramsey ha podido presentar la información básica, aunque de forma tremendamente sucinta, conservando todo lo posible el formato de la Loeb. Algunas notas al pie, no de la página, sino de cada traducción completan los datos. Aunque difícilmente se podría esperar otra solución por razones editoriales y a pesar de que, gracias al profundo conocimiento que tiene de estos textos, Ramsey haya conseguido normalmente proporcionar en estos reducidos comentarios los conocimientos básicos, son muchos los pasajes en los que el lector tendrá que acudir a otros libros si quiere estar al tanto de las razones de la crítica para establecer una

${ }^{35}$ K. Büchner, Sallust, Heidelberg 1982², 420-1 nn. 183-4, que aduce Plu. Sert. 22.10-1. Es verdad que poco más adelante no hay mucho que explicar de 145 o 146.

${ }^{36}$ Son probablemente dos descuidos: en general el discurso, como el resto del comentario, está soberbiamente resuelto, véanse e. g. las pp. 204-5.

${ }^{37}$ G. Linker, Emendationen zu Sallust, Wien 1854, 27-8, cf. Plut. Sert. 3.7. 
hipótesis, por ejemplo, o si pretende tener todas las fuentes antiguas que aclaran un texto.

La introducción consta de un prefacio, dedicado a pasar revista a las obras más útiles con las que ha contado el editor y a los agradecimientos, y una pequeña introducción que, tras remitir al lector al primer volumen para la vida y obra de Salustio ${ }^{38}$, está dedicada a la trasmisión del texto, con unas acertadas palabras sobre lo que se puede saber de la estructura original de la obra, y a presentar la edición (XX-XXV). En las referencias se pueden encontrar las abreviaturas, las ediciones de los testimonios indirectos ${ }^{39}$, una bibliografía sucinta y las siglas. En conjunto la bibliografía es una excelente selección: faltan muchos artículos de crítica y discusión de fragmentos concretos ${ }^{40}$, pero la verdad es que Ramsey, probablemente por razones de espacio, no suele mencionarlos después en el texto o la traducción, por lo que no hubiera tenido sentido citarlos aquí.

La traducción en general es excelente. Ramsey no solo ha procurado trasmitir exacta y cuidadamente el original, sino que además lo ha hecho en un lenguaje ágil y actual. Comparada con la de McGushin, por ejemplo, es claramente mejor, mucho más cercana al texto de Salustio y comúnmente más acertada. Posiblemente se podría alegar alguna cuestión discutible ${ }^{41}$, pero normalmente no ha lugar a controversias graves.

Quizá se puedan señalar más salvedades con respecto a sus preferencias editoriales, y concretamente a la colocación de cada uno de los fragmentos. Sin pretender repasar la ordenación completa, da la impresión de que el optimismo de Maurenbrecher (y Kritz) de poder situar la mayoría de los textos en un lugar de la historia no se ha corregido suficientemente. El escepticismo que ya preconizaba La Penna en 1963 quizá se debería haber

${ }^{38}$ Cf. n. 25.

${ }^{39}$ Hubiera hecho bien, por cierto, en seguir el sistema de Reynolds de citar la edición de Arusiano Mesio de Della Casa por el número del lema, no por la página, porque esta tiene por un lado el texto, por otro la traducción y por otro el comentario.

${ }^{40}$ Solo como ejemplo entre la crítica italiana, además del volumen de S. Boldrini et al., eds., Atti del convegno 'Gli storiografi latini tramandati in frammenti' (Urbino, 9-11 maggio 1974), Studi Urbinati di Storia, Filosofia e Letteratura 49.1, Urbino 1975, véase P. Frassinetti, "Su alcuni frammenti delle Historiae di Sallustio", Athenaeum 40, 1962, 93-102; O. Pecere, "Su alcuni frammenti delle Historiae di Sallustio", SIFC 41.1-2, 1969, 61-80; "Un frammento di Sallustio tra propaganda politica e polemica storiografica", RFIC 104, 1976, 399-417; "Note sui frammenti di Sallustio", SIFC 50.1-2, 1978, 131-60; G. Garbugino, "Il I libro delle Historiae di Sallustio in Nonio Marcello", Studi Noniani 5, Genova 1978, 39-94; "Note al II libro delle Historiae di Sallustio", Studi Noniani 11, Genova 1986, 31-58; "Due note sallustiane”, Studi Noniani 12, Genova 1987, 61-5; “Tre note alle Historiae di Sallustio”, en S. Feraboli, ed., Mosaico. Studi in onore di Umberto Albini, Genova 1993, 83-6; "La posizione politica di Sallustio", en R. Uglione, ed., Atti del Convegno Nazionale di Studi "Scrivere la storia nel mondo antico" (Torino 3-4 maggio 2004), Alessandria 2006, 111-40.

${ }^{41}$ No sé si está totalmente acertado, por ejemplo, el final de $1.12(=12 \mathrm{M})$ o el de 2.101 (= $105 \mathrm{M}$, p. 207), un texto, por otra parte, muy castigado e inseguro; tampoco parece adecuada la traducción de Lati (e. g. p. 43), demasiado perifrástica. 
llevado más lejos de lo que este y Ramsey han hecho en estos últimos años. Así, por ejemplo, se envía con toda lógica a los fragmentos de localización incierta $4.34 \mathrm{M}$-diu noctuque laborare, festinare (inc. 9 Ramsey)-, de acuerdo una vez más con McGushin, pero mantiene 4.35 como $4.24-$ frigida nocte ( $=4.29 \mathrm{McGushin})$ - cuando hay incluso más razones para retirarlo del libro. Resulta igualmente inexplicable que mantenga $2.33 \mathrm{M}-$ copiis integra (=2.33 McGushin / 2.32 Ramsey)-, quizá por tradición, pues también lo retuvo Dietsch, pero está claro que el significado exacto de copiis en $\tan$ breve fragmento se nos escapa ${ }^{42}$. Es posible que hayan pesado en estos lugares y en otros las preferencias de McGushin. También $3.75 \mathrm{M}$-quem trans stagnum omnis usque ad flumen- se puede interpretar como una descripción o como una escena de guerra o casi como cualquier otra cosa y además está muy corregido: debería haber figurado entre los textos incertae sedis $^{43}$. En estos casos y en otros se manifiesta una excesiva confianza en nuestra capacidad para reconstruir Historias ${ }^{44}$.

Aunque Ramsey en general ha efectuado una ordenación personal de los fragmentos, es deudor, como parecía lógico, de las de Maurenbrecher y, sobre todo, de McGushin, con el que coincide en mayor medida que con el primero ${ }^{45}$.

Aunque las correcciones de Ramsey en el texto no son frecuentes, interviene en algunos momentos muy concretos. Así en $1.52(=1.60 \mathrm{M})$ suple <lubidine> al final del fragmento a partir sin duda del cupidine de Maurenbrecher (1893, 28-9): aunque fonéticamente más próximo al contexto, no es más verosímil. Poco más adelante, en el $1.54(=1.62 \mathrm{M})$, añade el praenomen $\langle M$. $>$ ante Octavius, puesto que a continuación se encuentra $Q$. Caepio ${ }^{46}$. No es descabellado, pero tampoco necesario.

El volumen se completa con una traducción de las Cartas a César atribuidas a Salustio precedidas de una pequeñísima introducción y una nota sobre el texto. El formato aquí es el de una edición normal de la Loeb y Ramsey ha hecho un trabajo solvente, con una buena versión y las notas adecuadas. Para el resto de las obras del corpus salustiano, las invectivas, que no aparecen aquí, se remite al lector a otro volumen de la colección dedicado a Cicerón a cargo de Shackleton Bailey ${ }^{47}$.

${ }^{42}$ La Penna 1963 (n. 1), 34.

${ }^{43}$ Pero McGushin lo mantiene como 3.55 y Ramsey como 3.95. De Brosses (1777 —n. 8-, II, 107 y 228) y Kritz (1853 - n. 10-, 212) pensaron que se refería al cerco de Cícico (App. Mith. 72); McGushin (1994 -n. 22-, II, 108) de acuerdo con Maurenbrecher (1893 - n. 11-, 141) lo relaciona con los habitantes del lago Meótide, cf. Mela 1.114; Str. 11.2.4; Plin. nat. 6.19.

${ }^{44}$ R. Syme, Sallust, Berkeley-Los Ángeles 1964, 179.

${ }^{45}$ Por poner algunos ejemplos, toma de Maurenbrecher la secuencia de los fragmentos 71-83 del libro II (= 84-96 M) -cf. McGushin 1992 (n. 22), I, 247-50-, pero son muchísimas más las coincidencias con este último; véase, por ejemplo, 3.38-65 de Ramsey (60-87 Mc).

${ }^{46}$ Ya Dietsch - 1859 (n. 10), II, 19- había propuesto $\langle G n\rangle$.

${ }^{47}$ D. R. Shackleton Bailey, Cicero. Letters to Quintus and Brutus. Letter to Octavian. 
Las inevitables erratas, más disculpables si cabe en un libro tan complejo, están reducidas al mínimo y no entorpecerán seguramente al lector interesado ${ }^{48}$. Ramsey, por otro lado, corrige tácita y elegantemente algunos errores de sus predecesores ${ }^{49}$.

Las divergencias con la edición de Maurenbrecher (pp. 529-36) prueban por una parte la distancia de la crítica actual con respecto al sabio alemán y la independencia de criterio de Ramsey. Tras las inexcusables concordancias con Maurenbrecher y McGushin, se encuentran unos excelentes índices de fuentes de fragmentos y de nombres, con aclaraciones sobre su naturaleza tras el lema y, en el caso de las personas, remisión a Pauly-Wissowa. Igual que el primer volumen, está este ilustrado con unos magníficos mapas.

Con toda sinceridad, la edición en la Loeb no podía haberse resuelto con mejores resultados. Ramsey ha realizado una labor prodigiosa de concisión y selección de los datos esenciales y ha efectuado una revisión completa de la ordenación de los fragmentos. Evidentemente esto último hubiera estado mejor en otro tipo de libro en el que hubiera cabido una discusión exhaustiva de la crítica y defensa de las preferencias del editor, pero este precioso volumen, sin ninguna duda, figurará con todo merecimiento entre lo imprescindible y lo mejor de la bibliografía salustiana.

Buen año el 2015 para las Historias de Salustio.

JUAN Martos

Universidad de Sevilla

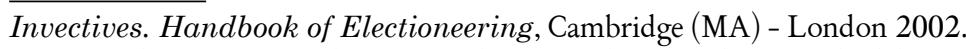

${ }^{48}$ En el 1.133 aparece el correspondiente 113 de McGushin cuando debería ser también 133, y se repite en las tablas de correspondencias. Véase was was en p. 227; The the (p. 19); Numanita (p. 189); Rylands (p. 399), probablemente por Robert, o cenes por censes (notas de p. 236). En p. 305 debería aparecer 1.114 en la cita de Mela; en p. 354, GLVI (y no 2). Falta el asterisco en 4.58 (p. 356) y 81 Mc en 2.85 (p. 190 y 191).

${ }_{49}$ Así, Eneida 1.640 (Maurenbrecher) en pág. 84 por 2.640. Al mismo editor se debe la errata 450 de $G L$ en $4.79 \mathrm{M}(=4.70 \mathrm{R})$ por 456 (p. 376). 